\title{
In-situ Observation and Characterization of Structural Evolution in a Phase- Change Memory Device by TEM-STM
}

\author{
Dongkyu Cha ${ }^{1}$, S.Y. Park ${ }^{1}$, Su Jin $\mathrm{Ahn}^{2}$, H. Horii ${ }^{2}$, D. H. Kim², Y. K. Kim², S.O. Park ${ }^{2}$, U In Jung ${ }^{2}$, \\ Moon J. Kim ${ }^{1}$, Jiyoung Kim ${ }^{1 *}$ \\ ${ }^{1}$ Dept. of Materials Science and Engineering, University of Texas at Dallas, Texas, 75080, U.S.A. \\ ${ }^{2}$ Memory Division, Semiconductor Business, Samsung Electronics Co, Hwasung, 445-701, Korea
}

Phase-change memory (PCM) devices are attractive prospects as the next generation of nonvolatile memory devices because of their high operating speed, endurance, and scalability [1]. In order to understand the mechanism behind the switching behavior of PCM devices, TEM analysis with laser annealing and thermal heating has been applied [2]. However, the phase transformation mechanism due to the electric field effect or the cell geometry effect cannot be explained because of the limitations of current conventional microscopy characterization methods. In this study, we demonstrate in-situ observation and characterization of a PCM device using specialized TEM-STM holders which provide in-situ TEM analysis and electrical characterization simultaneously.

To characterize and observe the device via TEM-STM holder, the sample was prepared by focused ion beam (FIB) with lift-out methods which provided site-specific sample preparation by using a nanomanipulator. To minimize $\mathrm{Ga}$ ion damage and contamination, a low accelerating voltage and beam current $(5 \mathrm{kV}, 0.5 \mathrm{pA})$ was used for the final thinning of the sample. The sample was attached to an $\mathrm{Au}$ wire holder and characterized in-situ via TEM as shown in figure 1. For probing the sample, a $\mathrm{W}$ tip is attached to a piezo-driven nanomanipulation stage on the TEM-STM holder for precisely controlled probing along all three axes. An Agilent 8110A pulse generator and a Keithley 4200 parameter analyzer were connected to the STM probe for applying programmed pulses and measuring device resistance.

The sample, which is programmed as an amorphous cell with $1 \mathrm{M} \Omega$, was investigated to compare the threshold voltage $\left(\mathrm{V}_{\text {th }}\right)$ in-situ and ex-situ. Under the current sweep test in the TEM-STM holder, the $\mathrm{V}_{\text {th }}$ was about $1.8 \mathrm{~V}$ as shown in figure 2 (a), which corresponds to the write voltage measured by a conventional probe station using wafer level memory devices. To observe the reversible switching behavior of the device, programmed pulses (figure 2 (b)) which have different quenching speeds (10ns: amorphization, 600ns: crystallization) are applied. Afterwards, the $\mathrm{Ge}_{2} \mathrm{Sb}_{2} \mathrm{Te}_{5}(\mathrm{GST})$ cell is reversibly changed depending upon the pulse condition. From the real time TEM observation, it is found that slow quenching crystallization enhances grain growth rather than the nucleation mechanism as shown in figure 2 (c). This result is a step towards understanding the fundamentals of PCM devices on behalf of reliability and scaling down enhancement.

References:

[1] Y. H. Shih, et al., IEDM Tech. Dig., pp. 207-210, 2008.

[2] S. A. Song et al., Ultramicroscopy, 108, pp. 1408-1419, 2008.

[3] We acknowledge financial support through the System IC 2010 program by MKE in Korea. 
(a)

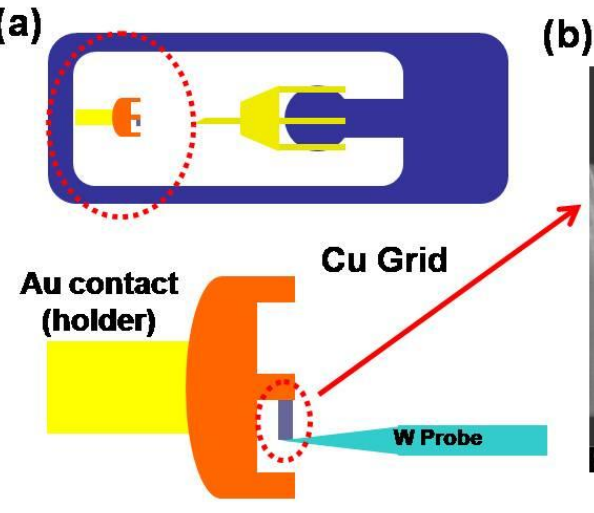

(b)

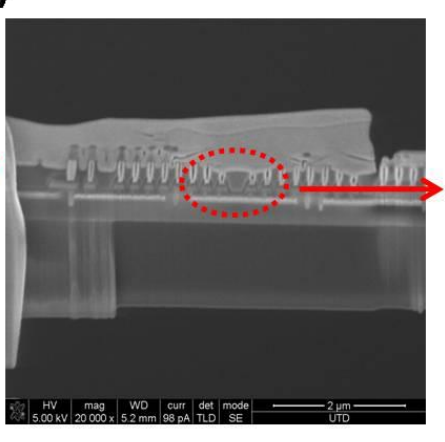

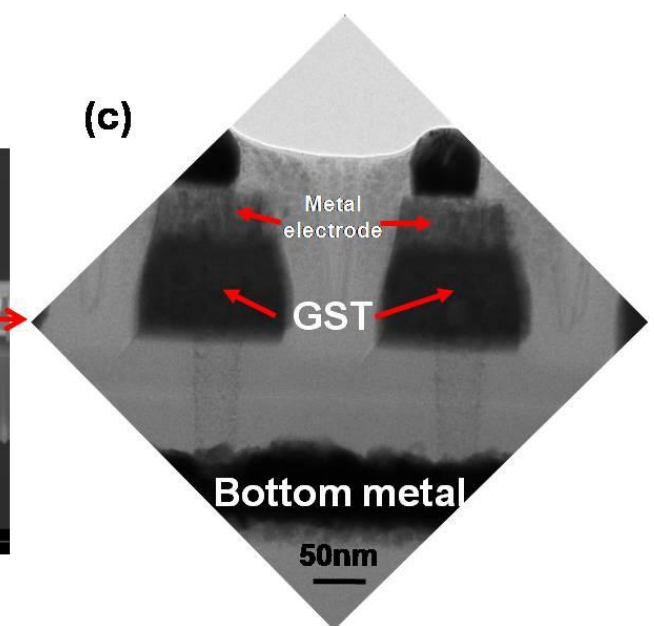

Figure 1. (a) Schematic diagram of TEM-STM holder and (b) SEM image of PCM device after FIB milling. (c) TEM image showing the structure of the PCM device. W tip probes the cylindrical metal contact on top of the metal electrode.

(a)

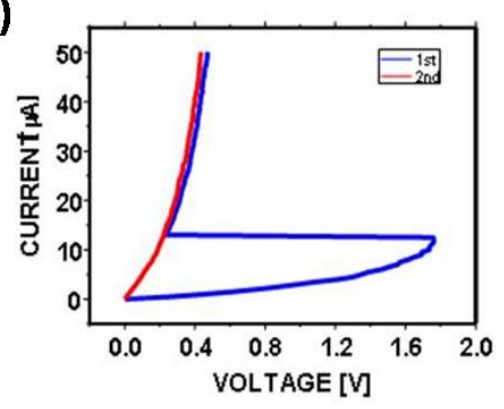

(b)

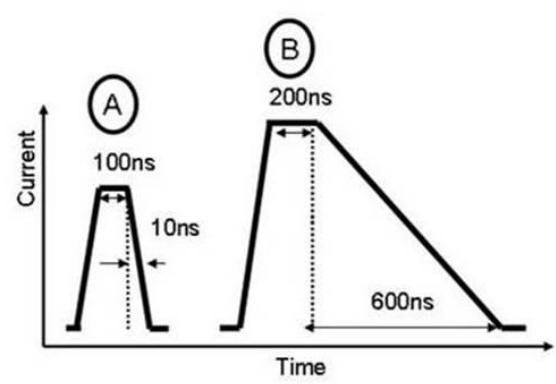

(c)
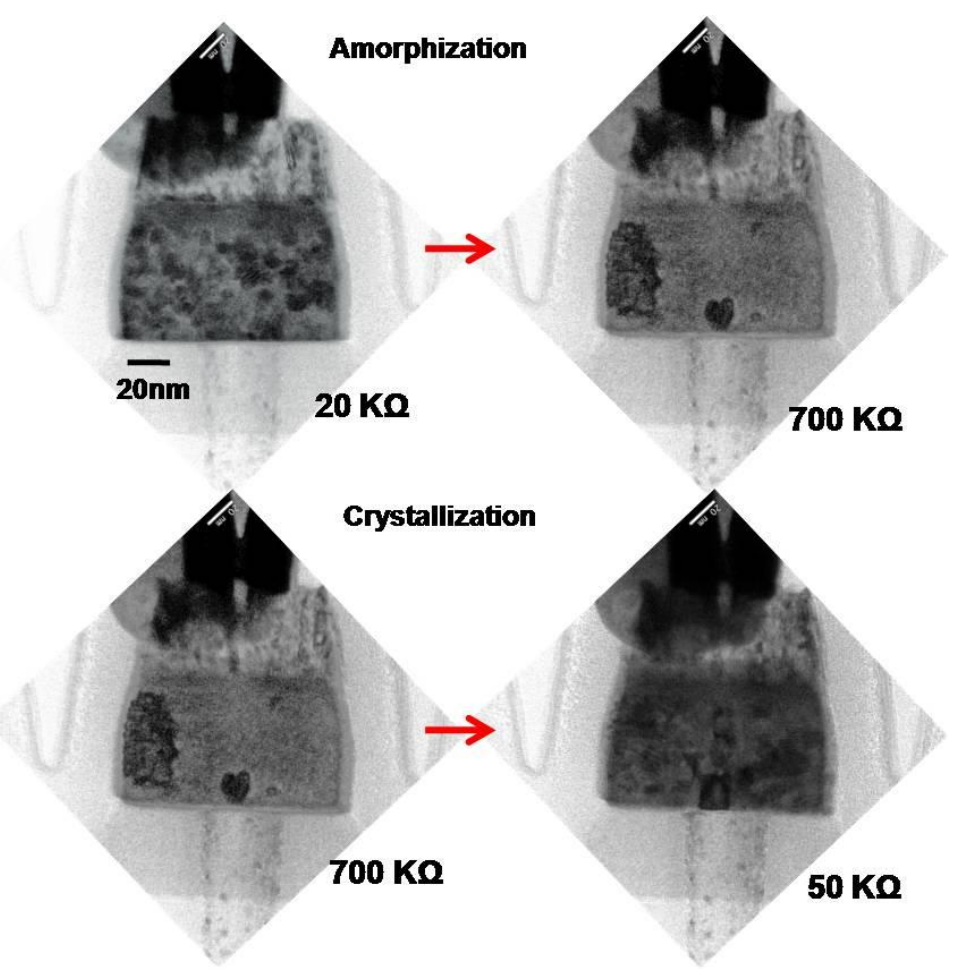

$700 \mathrm{~K} \Omega$

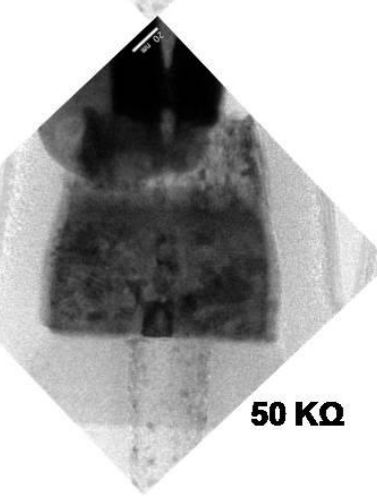

Figure 2. (a) I-V curves by applying current sweep. From the result, $\mathrm{V}_{\text {th }}$ and negative differential resistance was confirmed. (b) Schematic diagram of programming pulse shape. (c) TEM images for reversible switching of PCM device. Programmed pulses were applied to amorphize and crystallize the GST cell. The height and cooling speed of pulses for amorphization are $2.4 \mathrm{~V}$ (low current state) and $10 \mathrm{~ns}$, respectively. For crystallization, a pulse with $2.0 \mathrm{~V}$ (high current state) and $600 \mathrm{~ns}$ was applied. 